\title{
Diabetic Retinopathy Recognition using Enhanced Crow Search with Levy Flight Algorithm
}

\author{
Arun T Nair \\ KM C T College of Engineering \\ Manassery, Kerala, India \\ aruntnair8@gmail.com
}

\author{
Muthuvel K. \\ Noorul Islam University \\ Thuckaly, Tamil Nadu, India
}

\begin{abstract}
This paper aims to introduce an improved model for Diabetic Recognition (DR) recognition. Accordingly, the proposed model is executed under two stages, the initial one is the blood vessel segmentation and next step is the DR recognition. Using tophat by reconstruction of red portions in the green plane image, the two thresholds binary images are obtained in vessel segmentation. The areas that are found similar to two binary images are extracted as the main vessels. Additionally, the residual pixels in both the binary images are integrated in order to form a vessel sub-image i.e. facilitated to a classification of Gaussian Mixture Model (GMM). As a result, the complete pixels in the sub-image that are classified as vessels are amalgamated with the main vessels to obtain the segmented vasculature. Moreover, from the segmented blood vessel, the extraction of GLRM and Gray-Level Co-Occurrence Matrix (GLCM) features is performed that are subsequently classified by exploiting Neural Network. To enhance the accurateness, training is performed using Enhanced Crow Search with Levy Flight (ECS-LF) algorithm, so the error among actual output and predicted must be least.
\end{abstract}

Keywords: Diabetic Retinopathy; Neural Network; Feature Extraction; Optimization; Levy Flight

\begin{tabular}{ll} 
Nomenclature & \\
\hline Abbreviations & Descriptions \\
\hline DR & Diabetic retinopathy \\
HPF & High-Pass Filtering \\
GMM & Gaussian Mixture Model \\
NN & Neural Network \\
NPDR & Nonproliferative \\
PDR & Proliferative \\
IRMA & Intraretinal Microvascular Abnormality \\
STZ & Streptozotocin \\
Mfn2 & Mitofusin 2 \\
DAP & Dynamic Awareness Probability \\
PDR & Proliferative Diabetic Retinopathy \\
ML & Machine Learning \\
FBG & Fasting Blood Glucose \\
CNN & Convolutional Neural Network \\
ReLU & Rectified Linear Activation Unit \\
CSA & Crow Search Algorithm \\
RDR & Referable Diabetic Retinopathy \\
AP & Awareness Probability \\
\hline
\end{tabular}

\section{Introduction}

Generally, DR is one of the most difficult of diabetes and it is the reason for blindness in working-age people globally [18]. Initially, DR is represented as a microvascular difficulty of diabetes and it is categorized as PDR of NPDR or in attendance with neovascularization. Nevertheless, there is growing proof, which all cells resident in the retina is pretentious. Hence, DR is as well considered to be a neurodegenerative infection besides a microvascular difficulty. One of the most general manifestations of NPDR is augmented vascular permeability, microaneurysms, hemorrhages, intraretinal IRMA, and retinal capillary non-perfusion. By neovascularization, the proliferative DR is considered on the retina and subsequent vitreous surface, which tends to retinal detachment. 
DR is considered as the well-known ailments between the diabetic patients and they can prevent from loss of vision if the disease is analyzed at the previous phase. The patient has to be estimated once the disease is analyzed for every six months to recognize the improvement of the disease [15]. An efficient algorithm to identify and categorize the fundus images will be obliging for the ophthalmologist to a superior scope in eliminating the vision defeat because of the DR. In the study of image applications researchers have introduced numerous methods to assist accurate DR diagnosis. Moreover, the pattern of the human eye comprises optic nerves and disc. Classification and detection of DR can be performed by images segmentation of the sections of the elements from the fundus image or by probing the fundus image for the incidence of lesions, hemorrhages, microaneurysms, exudates, and so forth.

Latest developments in ML assure to help the right to use the DR screening considerably and to get better diagnosis precision. Systems developed by exploiting these algorithms (indicated as methods) have shown specialist-level accurateness while diagnosing DR [16]. On the other hand, in clinical settings, the effect of these algorithms is not well implicit. In a computer-assisted diagnosis setting earlier efforts to exploit machine learning algorithms have faced many confront, such as both under reliance and overreliance. If the computer-aided diagnosis system has the ability to elucidate its predictions, few of aforesaid drawbacks can be evaded [17]. In recent times, numerous developed elucidation algorithms concentrate on producing human-interpretable descriptions of how forecasting is done and might be an influential tool to help physicians inferring medical images, and retina images.

This main contribution of this paper is to propose an ECS-LF algorithm for DR recognition that is done under two phases, such as DR recognition and blood vessel segmentation. For that reason, from the segmented blood vessels, the feature extraction of GLRM and GLCM that are subsequently classified by exploiting NN. Furthermore, training of NN is performed using a novel algorithm named ECS-LF. One of the major intentions of the proposed DR recognition model is to reduce the error between the actual classification and predicted output.

\section{Literature Review}

In 2019, Asieh Naderi et al [1], studied detection malformation in visual function and vascular seepage in the STZ-persuaded diabetic rats subsequent to a minimum time. Moreover, they have exhibited the relentless retinal became different might be identified in STZ, which persuade diabetic rat in maximum time series of diabetes. Few modifications comprising vitreous neovascularization emotionally involved to the vitreoretinal traction and retina were related to the modifications, which happen in human proliferative DR. Tractional modifications might occur because of the pre-retinal membrane pattern which applies tractional forces based on the retina and drags it away from the layer beneath.

In 2019, Arul J. Duraisamy et al [2], examined the task for the fusion of the mitochondrial in the advancements of DR, and exemplify the molecular method dependable for Mfn2 suppression. By exploiting human retinal endothelial cells, influenced for Mfn2, the task of combination in mitochondrial functional and structural harm in diabetes was investigated. In diabetic milieu, the molecular model of its repression was estimated by examining Mfn2 advertiser DNA methylation, and verified by exploiting pharmacological and molecular inhibitors of DNA methylation.

In 2019, Ugur Gurlevik et al [3], examined the vitreous and serum levels of resistin in patients with the PDR and to contrast those with age-matched control themes. The investigation comprises two groups: a control group of 22 and 45 eyes with PDR. Moreover, all eyes undergo vitrectomy surgery. In blood samples, the lipid profile, HbA1c, FBG, and resistin levels were examined of all subjects and evaluated entire ophthalmological assessments. During vitrectomy resistin and surgery levels, vitreous samples were gathered from both groups were examined in those samples.

In 2019, T. Shanthi and R.S. Sabeenian [4], developed on the DR fundus images classification in accordance with the disease severity exploiting CNN with the function of appropriate ReLU layers, pooling, and softmax to attain a maximum level of accurateness. The performance of the developed model was examined by exploiting Messidor database.

In 2018, Xianglong Zeng et al [5], worked on DR, which was a significant reason for blindness global. Nevertheless, DR was ard to recognize in first phases and the diagnostic process might be time-utilizing even for knowledgeable specialists. Hence, a computer-assisted diagnosis algorithm on the basis of the deep learning model was proposed to automatedly diagnose the RDR through the classification of color retinal fundus snaps into two grades. Here, a new CNN algorithm with Siameselike framework was trained with transfer learning model. In contrast with preceding studies, the developed model identifying binocular fundus images as inputs and studies their association to assist generating prediction.

In 2018, Yunlei Sun [6], presented the CNN model to 1-dimensional irrelevant data sets and resolve the issue of how to perform 1-dimensional unrelated data convolution. Moreover, the CNN model was integrated with the BN layer to avoid the gradient dispersion, accelerate the training speed and enhance 
the accurateness of the model. Moreover, the proposed model integrates a rate of adaptive learning model in addition to optimize the algorithm.

\section{Proposed Diabetic Recognition Model}

\subsection{Proposed Model}

The diagrammatic representation of the proposed model on the basis of the DR diagnosis is shown in Fig. 1. The proposed model is done on 2 phases, the initial one is the blood vessel segmentation and next step is the DR recognition. The developed segmentation of vessel model is performed for each color fundus image in three stages. In the first phase, two thresholded binary images are achieved; initial one is by $\mathrm{HPF}$ and subsequent one is by tophat. It is done by reconstructing the red segments in green plane image. The areas which are found similar to the two binary images are obtained as the main vessels, and the remaining against pixels in both binary images are amalgamated to create a sub-image vessel. Consequently, in the following phase, pixels originate in the vessel sub-image are subjected to a classification of GMM to identify the vessel. In the sub-image the complete pixels, that are classified, and combined with the main vessels for obtaining the vasculature segmentation. On the basis of the resolution for the fundus images, the segmentation of vessels is additionally enhanced to create definite regarding the superior vessel segmentation correctness. In additional from the blood vessels segmentation, the features from the GLRM and GLCM are extracted that are subsequently classified by exploiting improved NN. Besides, to enhance the precision, the NN weights are optimized using the ECS$\mathrm{LF}$ as a training model. Furthermore, this work contributes to performing the recognition of DR with the contribution of reducing the error among the actual and predicted output. Hence, the existence of diabetes is recognized efficiently from the developed recognition of DR algorithm.

\subsection{Segmentation Phase}

Let us assume the input fundus image as IM that is given to segmentation of blood vessel procedure. Moreover, the developed segmentation of vessel model is done in three stages. In the first phase, two binary images are obtained: the primary phase is performed using HPF and next is performed using the reconstruction of tophat.

An HPF is a filter, which permits maximum frequencies on the form, though; it diminishes frequencies, which are least than the cut-off frequency. Chiefly, in the frequency domain sharpening is considered as a high pass function. There are several benchmark functions of HPF like Ideal, Gaussian HPF, and Butterworth. All HPF is frequently shown by its relationship to the LPF. Eq. (1) represents the HPF filter formulation.

$$
\mathrm{HPF}=1-\mathrm{LPF}
$$

Hence, the maximum-frequency image obtained from HPF and it is indicated by $\mathrm{IM}_{\mathrm{H}}$.

Top Hat by reconstruction: Arithmetical organization is a vital idea founded on "set theory" which is extensively exploited in pattern recognition and optical signal processing and pattern recognition [7]. Generally, operators in morphological are showed based on the original image $p(x, y)$ and structuring element $\mathrm{S}(\mathrm{u}, \mathrm{v})$. $(\mathrm{u}, \mathrm{v})$ and $(\mathrm{x}, \mathrm{y})$ denotes coordination of pixels in $\mathrm{S}$ and $\mathrm{p}$, respectively. The basic morphological operator is dilation and erosion. Erosion $(\sigma)$ and dilation $(\Delta)$ of $\mathrm{S}(\mathrm{u}, \mathrm{v})$ by $\mathrm{p}(\mathrm{x}, \mathrm{y})$ are stated in eq. (2) and eq. (3).

$$
\begin{gathered}
\Delta_{\alpha}(\mathrm{h})(\mathrm{x}, \mathrm{y})=\max _{\mathrm{u}, \mathrm{v}}(\mathrm{p}(\mathrm{x}-\mathrm{u}, \mathrm{y}-\mathrm{v})+\mathrm{S}(\mathrm{u}, \mathrm{v})) \\
\sigma_{\alpha}(\mathrm{h})(\mathrm{x}, \mathrm{y})=\min _{\mathrm{u}, \mathrm{v}}(\mathrm{h}(\mathrm{x}+\mathrm{u}, \mathrm{y}+\mathrm{v})-\mathrm{S}(\mathrm{u}, \mathrm{v}))
\end{gathered}
$$

The geodesic dilation of the dimension $m$ of the marker image $\mathrm{p}$ regarding the mask image $\mathrm{h}$ stated by $\Delta_{\mathrm{h}}^{(\mathrm{m})}$ is denoted as "the pixel-wise least among $\mathrm{p}$ and the dilation of $\mathrm{h}$ exploiting structuring element S, iteratively", as indicated in eq. (4) and (5).

$$
\begin{aligned}
& \Delta_{\mathrm{h}}^{(\mathrm{m})}(\mathrm{p})=\Delta_{\mathrm{h}}^{(1)}\left(\Delta_{\mathrm{h}}^{(\mathrm{m}-1)}(\mathrm{p})\right) \\
& \Delta_{\mathrm{h}}^{(1)}(\mathrm{p})=\min \left(\mathrm{h}, \Delta_{\mathrm{S}}^{(1)}(\mathrm{pd})\right)
\end{aligned}
$$

The dimension $\mathrm{m}$ for the geodesic dilation of $\mathrm{p}$ regarding $\mathrm{h}$ stated in $\sigma_{\mathrm{h}}^{(\mathrm{m})}$ is defined as "the pixelwise maximum among $\mathrm{p}$ and the dilation of $\mathrm{h}$ exploiting structuring element $S$, iteratively" as indicated in eq. (6) and (7).

$$
\sigma_{\mathrm{h}}^{(\mathrm{m})}(\mathrm{p})=\sigma_{\mathrm{h}}^{(1)}\left(\sigma_{\mathrm{h}}^{(\mathrm{m}-1)}(\mathrm{p})\right)
$$




$$
\sigma_{\mathrm{h}}^{(1)}(\mathrm{p})=\max \left(\mathrm{h}, \sigma_{\mathrm{S}}^{(1)}(\mathrm{p})\right)
$$

$\mathrm{RC}_{\mathrm{h}}^{\sigma}$ denotes the reconstruction of $\mathrm{h}$ from $\mathrm{p}$ using dilation and it is described as "the iterative geodesic dilation of $\mathrm{p}$ exploiting $\mathrm{h}$ until the stability" as indicated in eq. (8).

$$
\mathrm{RC}_{\mathrm{h}}^{\sigma}(\mathrm{p})=\sigma_{\mathrm{h}}^{(\mathrm{s})}(\mathrm{p}), \text { where } \sigma_{\mathrm{h}}^{(\mathrm{s})}(\mathrm{p})=\sigma_{\mathrm{h}}^{(\mathrm{s}+1)}(\mathrm{p})
$$

The opening reconstruction is described as "the reconstruction of an image $p$ from the erosion of $p$ exploiting structuring element $\mathrm{S}$ “, that is based upon the dilation reconstruction as denoted in eq. (9).

$$
\mu_{\mathrm{S}}^{\mathrm{RC}}(\mathrm{p})=\mathrm{RC}_{\mathrm{p}}^{\Delta}\left(\sigma_{\mathrm{S}}(\mathrm{p})\right)
$$

Moreover, closing reconstruction is described as "the reconstruction of an image $\mathrm{p}$ from the dilation of $\mathrm{p}$ exploiting structuring element $\mathrm{S}$ ", that is based upon the reconstruction of erosion as indicated in eq. (10).

$$
\kappa_{\mathrm{S}}^{\mathrm{RC}}(\mathrm{p})=\mathrm{RC}_{\mathrm{p}}^{\sigma}\left(\Delta_{\mathrm{S}}(\mathrm{p})\right)
$$

By reconstruction, the operators of top-hat, collectively with the white and black top-hat operators, where defined as "the difference among the image $\mathrm{p}$ and the consequence of opening or closing by reconstruction operator" is indicated in eq. (11) and (12) respectively.

$$
\begin{aligned}
& \operatorname{RWTH}(p)=p-\mu_{\mathrm{S}}^{R C}(p) \\
& \operatorname{RBTH}(p)=\kappa_{S}^{R C}(p)-p
\end{aligned}
$$

Hence the ensuing image obtained by this model is indicated by $I M_{T}$.

The areas widespread to $\mathrm{IM}_{\mathrm{H}}$ and $\mathrm{IM}_{\mathrm{T}}$ are obtained as main vessels, whereas the remaining pixels in both binary images are combined to create a sub-image vessel, indicated by $\mathrm{IM}_{\mathrm{V}}$.

\subsection{Gaussian Mixture Model}

In the following phase, the pixels $\mathrm{IM}_{\mathrm{V}}$ are developed as an input for the classification of GMM. GMM [8] is stated as the weighted sum of multiple Gaussian components that represent a density of a particular random variable. A discrete set of Gaussian operations in GMM allows a developed modeling capability. Moreover, the mathematical modeling of GMM is indicated in eq. (13), where $y$ indicate the random variable, covariance Matrix (CM), K denotes the maximum number of Gaussian elements and $\Theta$ denotes to the GMM constraints, that include mean vector $(\mu)$, and weight (wt $\geq 0)$ which assures $\sum_{\mathrm{i}=1}^{\mathrm{K}} \mathrm{wt}_{\mathrm{i}}=1, \mathrm{CM}\left(\mathrm{y} \mid \mu_{\mathrm{i}}, \Sigma_{\mathrm{i}}\right)$ is the Gaussian element density $\mathrm{i}$ as indicated in eq. (14), whereas DM represents the dimension of $\mathrm{y}$.

$$
\begin{aligned}
& \operatorname{CM}(\mathrm{y} \mid \Theta)=\sum_{\mathrm{i}=1}^{\mathrm{K}} \mathrm{wt}_{\mathrm{i}} \operatorname{CM}\left(\mathrm{y} \mid \mu_{\mathrm{i}}, \Sigma_{\mathrm{i}}\right) \\
& \operatorname{CM}\left(\mathrm{y} \mid \mu_{\mathrm{i}}, \Sigma_{\mathrm{i}}\right)=\frac{1}{(2 \pi)^{\frac{\mathrm{DM}}{2}}\left|\Sigma_{\mathrm{i}}\right|^{\frac{1}{2}}} \mathrm{e}^{-\frac{1}{2}\left(\mathrm{y}-\mu_{\mathrm{i}}\right) \Sigma_{\mathrm{i}}^{-1}\left(\mathrm{y}-\mu_{\mathrm{i}}\right)}
\end{aligned}
$$

Hence the classified fundus image using the GMM model is indicated by $\mathrm{IM}_{\mathrm{GMM}}$. Consequently, the areas related to the two binary images attained from tophat and HFPF and it is attained by reconstructing the main vessels. Additionally, the remaining pixels seen in both the binary images are merged to produce sub-image vessel and it is again classified by exploiting GMM classifier. Moreover, the complete pixels in the sub-image that are classified as vessels are united within the main vessels for obtaining the improved detection. Therefore, the last segmented main vessel is indicated $\mathrm{IM}_{\text {Final }}$. 


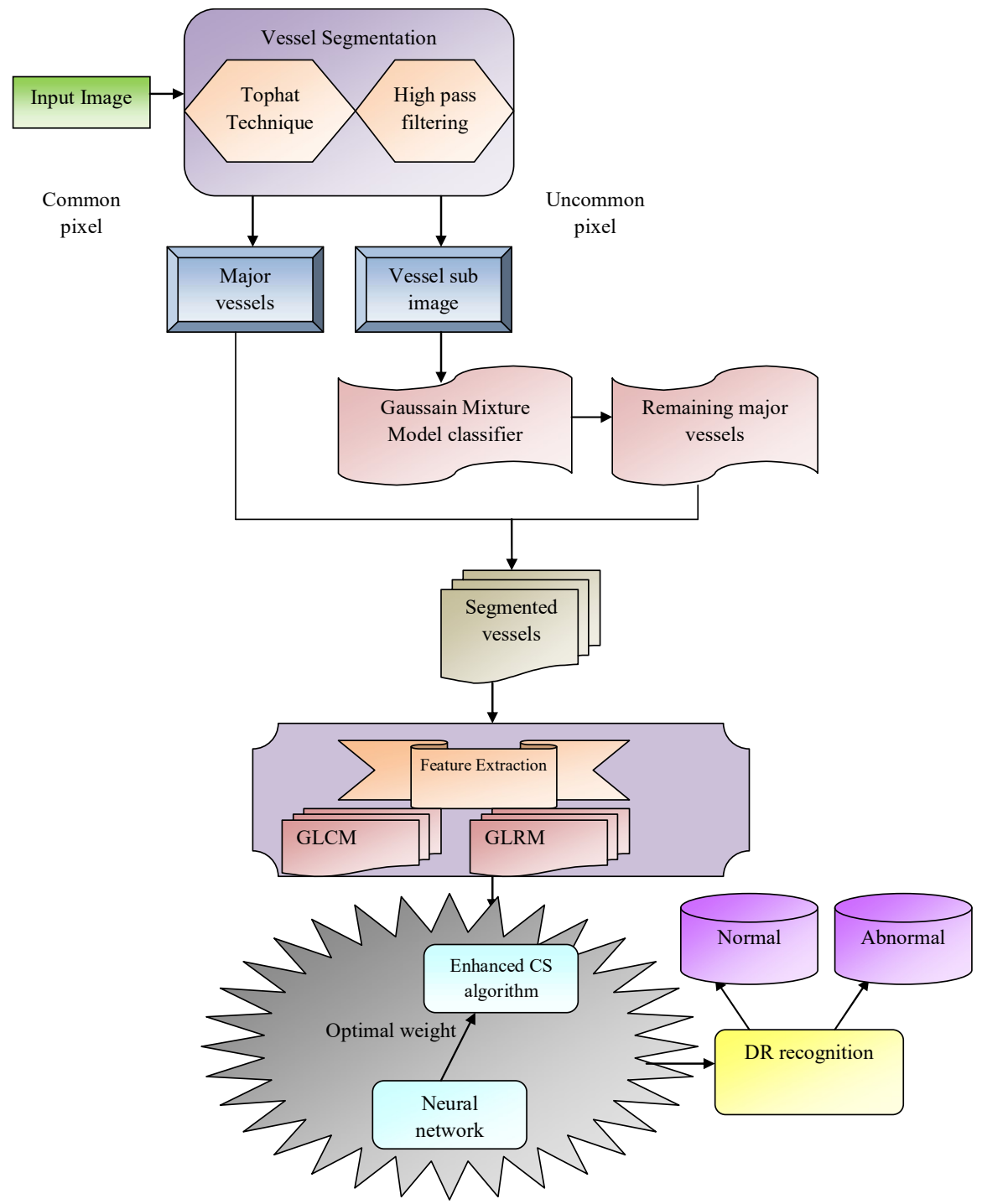

Fig. 1. System model of the proposed model

\section{Improved Neural Network for Feature Extraction}

On account of the resolution of the fundus image, the vessels segmentation is enhanced to assurance the precision of high segmentation of vessel. For that reason, from the blood vessels segmentation, the GLRM, and GLCM features, are extracted. The GLRM [10] and GLCM [9] features are extracted from the blood vessels segmentation. The concise definition of the GLRM and GLCM features

\subsection{Neural Network Model}

For classification purpose, the extracted features $\mathrm{IM}_{\mathrm{f}}$ are subjected to feed-forward NN classifier. The $\mathrm{NN}$ [11] is a classifier which attained the features as the input, and it is stated in eq. (15), whereas $\mathrm{NF}$ indicates the number of features.

$$
\operatorname{IM}_{\mathrm{f}}(\mathrm{n})=\left\{\mathrm{IM}_{\mathrm{f}_{1}}, \mathrm{IM}_{\mathrm{f}_{2}}, \ldots \ldots . . . \mathrm{IM}_{\mathrm{fNU}_{\mathrm{NU}}}\right\}
$$

As eq. (16), (17) and (18), the network model is denoted in that $\mathrm{i}$ indicates the hidden neuron, $\mathrm{n}_{\mathrm{h}}$ denotes the number of hidden neurons, $\mathrm{Wt}_{(\mathrm{Bi})}^{(\mathrm{H})}$ indicates to the bias weight to $\mathrm{i}^{\text {th }}$ hidden neuron, $\mathrm{n}_{\mathrm{h}}$ denotes the number of output neurons, and $\mathrm{Wt}_{(\mathrm{im})}^{(\mathrm{o})}$ signifies the weight from $\mathrm{i}^{\text {th }}$ hidden neuron to $\mathrm{m}^{\text {th }}$ output neuron. In addition, $\mathrm{Wt}_{(\mathrm{Bm})}^{(\mathrm{o})}$ denotes to the bias weight to $\mathrm{m}^{\text {th }}$ output neuron, $\mathrm{Wt}(\mathrm{Hi})$ denotes the weight from $\mathrm{j}^{\text {th }}$ input neuron to $\mathrm{i}^{\text {th }}$ hidden neuron. The hidden output is stated in eq. (16), 
and general network output $\mathrm{NO}_{\mathrm{m}}$ is shown in eq. (17), in that AF indicates as the activation function $\mathrm{n}_{\mathrm{i}}$ denotes the number of input neurons. The error model indicates the absolute difference of predicted and actual output as stated in eq. (18) that needs to be reduced. In eq. (18), $\mathrm{NO}_{\mathrm{m}}$ indicates the actual output, and $\mathrm{NO}_{\mathrm{m}}$ indicates the predicted output. The weight $\mathrm{Wt}_{1}=\left\{\mathrm{Wt}_{(\mathrm{Bi})}^{(\mathrm{G})}, \mathrm{Wt}_{(\mathrm{Bm})}^{(\mathrm{o})}, \mathrm{Wt}_{(\mathrm{ji})}^{(\mathrm{G})}, \mathrm{Wt}_{(\mathrm{im})}^{(\mathrm{o})}\right\}$ has to be tuned properly for mining the error. Fig 2 demonstrates the schematic diagram of the neural network model.

$$
\begin{aligned}
& e^{(G)}=A F\left(W t_{(B i)}^{(G)}+\sum_{j=1}^{n_{i}} W_{(j i)}^{(G)} \operatorname{IM}_{f}(n)\right) \\
& \left.N \hat{O}_{m}=A F\left(\mathrm{Wt}_{(\mathrm{Bm})}^{(\mathrm{o})}+\sum_{\mathrm{i}=1}^{\mathrm{n}_{\mathrm{h}}} \mathrm{Wt}_{(\mathrm{im})}^{(\mathrm{o})}\right)_{\mathrm{i}}^{(\mathrm{G})}\right)
\end{aligned}
$$

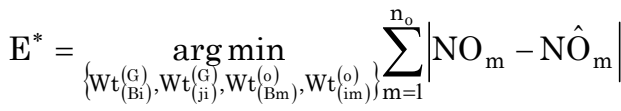

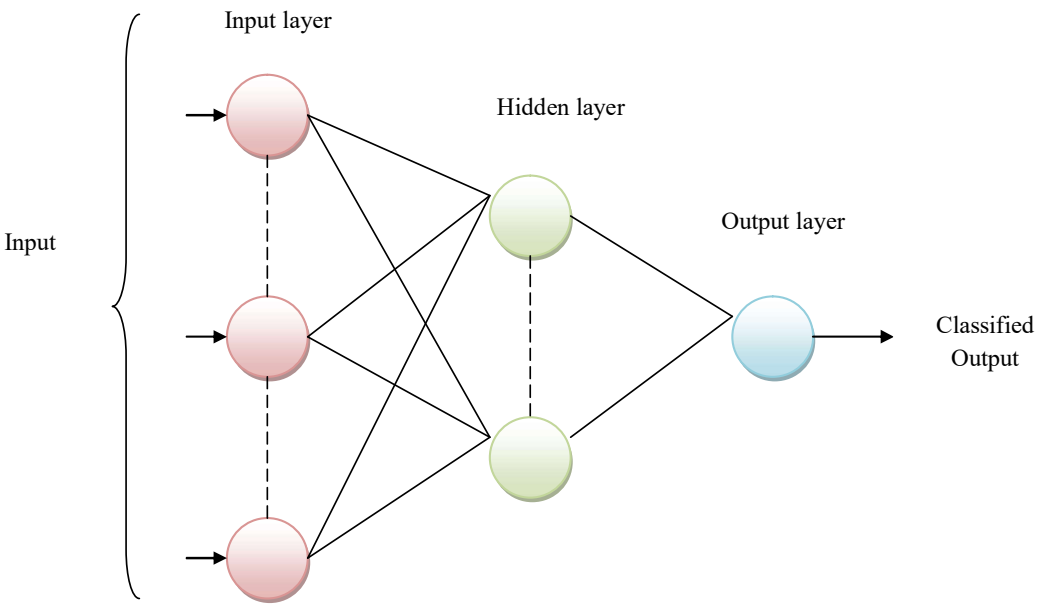

Fig. 2. Schematic diagram of neural network model

\subsection{Conventional CSA}

CSA is the latest metaheuristic algorithm [12] that is enthused on the intelligence behavior of crows [13] [14]. The process of CSA evolutionary imitates the behavior by crows of recovering and hiding additional food. As an algorithm on the basis of the population, the flock size is confirmed by $\mathrm{N}$ crows (individuals) that are of $\mathrm{n}$-dimensional with $\mathrm{n}$ as the dimension of the problem. The position $\mathrm{Y}_{\mathrm{i}, \mathrm{j}}$ of the crow $i$ in an exacting iteration $\mathrm{j}$ is denoted as eq. (19) indicates a probable solution for the issue:

$$
Y_{i, j}=\left\lfloor y_{i, j}^{1}, y_{i, j}^{2}, \ldots ., y_{i, j}^{n},\right\} i=1,2, \ldots ., N ; j=1,2, \ldots ., \max _{\text {iter }}
$$

In eq. (19) $\max _{\text {iter }}$ represents the maximum iterations in the procedure. Every individual (crow) is supposed to encompass the ability to memorize the optimal visited position $P_{i, j}$ to conceal food until the current iteration using eq. (20).

$$
P_{i, j}=\left\lfloor p_{i, j}^{1}, p_{i, j}^{2}, \ldots . . p_{i, j}^{n}\right\rfloor
$$

The crow $\mathrm{i}$ recognizes concerning the attendance of crow $\mathrm{k}$ and to defend its food, crow $\mathrm{i}$ intentionally obtains a random route. This behavior is imitation in CSA by the functioning of an arbitrary movement. The kind of behavior contemplated by every crow i is decided by an AP in an evasion.

The position of each is altered on the basis of two behaviors such as evasion and pursuit. A crow $\mathrm{k}$ pursues crow i with the reason to find out its concealed place. The crow $\mathrm{i}$ does not observe the attendance of the other crow; as a result, the reason for crow $\mathrm{k}$ is attained in pursuit.

Hence, the value of random $u_{i}$ uniformly distributed among zero and one is sampled. If $u_{i}$ is greater or equivalent than $\mathrm{AP}$, behavior one is exploited, else circumstances 2 is selected. 


$$
\mathrm{Y}_{\mathrm{i}, \mathrm{j}+1}=\left\{\begin{array}{l}
\mathrm{Y}_{\mathrm{i}, \mathrm{j}}+\mathrm{u}_{\mathrm{i}} \cdot \mathrm{fl}_{\mathrm{i}, \mathrm{j}} \cdot\left(\mathrm{P}_{\mathrm{k}, \mathrm{j}}\right) \mathrm{u}_{\mathrm{i}} \geq \mathrm{AP} \\
\text { random } \quad \text { O.W }
\end{array}\right.
$$

The parameter flight length $\mathrm{fl}_{\mathrm{i}, \mathrm{j}}$ denoted the magnitude movement from crow $\mathrm{Y}_{\mathrm{i}, \mathrm{j}}$ to the optimal position $\mathrm{P}_{\mathrm{k}, \mathrm{j}}$ of a crow $\mathrm{k}$, the $\mathrm{u}_{\mathrm{i}}$ is a random number with uniform distribution in the range $[0,1]$. Formerly the crows are adapted; their position is estimated and the memory vector is updated using eq. (22).

$$
P_{i, j+1}=\left\{\begin{array}{l}
F\left(Y_{i, j+1}\right) F\left(Y_{i, j+1}\right)<F\left(P_{i, j}\right) \\
P_{i, j} \quad \text { O.W }
\end{array}\right.
$$

In eq. (22), $\mathrm{F}(\bullet)$ indicates the objective model to be reduced.

\subsection{Proposed Enhanced CS Algorithm with Levy Flight}

The conventional CS algorithm has shown it's possible to discover the best solution for definite search spaces configurations [12]. Nevertheless, its convergence is not definite because of unsuccessful exploration of its search scheme. In the proposed ECS-LF algorithm, elements, the AP and the random movement, are rewritten.

The AP is replaced by a DAP that is a probability value modified using the fitness quality for every candidate solution. The exploit of probability parameters on the basis of the fitness values is effectively developed in the evolutionary algorithms [22]. Therefore, the DAP is computed using eq. (23).

$$
\mathrm{DAV}_{\mathrm{i}, \mathrm{j}}=0.9 \cdot \frac{\mathrm{F}\left(\mathrm{Y}_{\mathrm{i}, \mathrm{j}}\right)}{\omega \mathrm{W}}+0.1
$$

In eq. (23) $\omega \mathrm{W}$ indicates the worst fitness value observed till now. Presumptuous a minimization issue, this value is computed as pursues $\omega \mathrm{W}=\max \left(\mathrm{F}\left(\mathrm{Y}_{\mathrm{i}, \mathrm{j}}\right)\right)$.

Lévy flights are a kind of random walk that is experimented in various species as a foraging model [19]. It is the step size, which is inhibited by a heavy-tailed probability distribution typically referred to as Lévy distribution. Lévy Flights are highly efficient in exploring the search space than the uniform random distribution [20].

In the developed ECS-LF, the main purpose is to encompass a superior diversification on the search space. To imitate the evasion behavior, lévy flights are exploited rather than uniform random movements. Hence, a novel random position $\mathrm{Y}_{\mathrm{i}, \mathrm{j}+1}$ is produced adding together to the current position $\mathrm{Y}_{\mathrm{i}, \mathrm{k}}$ the calculated Lévy flight LF .

For LF to attain asymmetric Lévy stable distribution the Mantegna algorithm [21] is exploited. In Mantegna algorithm, the initial phase is to compute the step size $S_{i}$ using eq. (24).

$$
\mathrm{S}_{\mathrm{i}}=\frac{\mathrm{c}}{|\mathrm{d}|^{1 / \alpha}}
$$

In eq. (24), $\mathrm{c}$ and $\mathrm{d}$ represents $n$-dimensional vectors and $\alpha=\frac{3}{2}$. From the normal distribution, the elements of every vector $c$ and $d$ are sampled, which are categorized by exploiting eq. (25).

$$
\begin{aligned}
& \mathrm{c} \approx \mathrm{N}\left(0, \lambda_{\mathrm{c}}^{2}\right) \mathrm{d} \approx \mathrm{N}\left(0, \lambda_{\mathrm{d}}^{2}\right) \\
& \lambda_{\mathrm{c}}=\left\{\frac{\Gamma(1+\alpha) \sin (\pi \alpha / 2)}{\Gamma(1+\alpha) \sin (\pi \alpha / 2) \alpha 2^{(\alpha-1) / 2}}\right\}, \lambda_{\mathrm{d}}=1
\end{aligned}
$$

In eq. (25), $\Gamma(\cdot)$ signifies a Gamma distribution. Subsequent to attaining the value of $\mathrm{S}_{\mathrm{i}}$, the factor $\mathrm{LF}$ is computed using eq. (26).

$$
\mathrm{LF}=0.01-\mathrm{S}_{\mathrm{i}} \Theta\left(\mathrm{Y}_{\mathrm{i}, \mathrm{j}}-\mathrm{Y}_{\text {best }}\right)
$$

In eq. (26), the product $\Theta$ signifies the element-wise multiplications, $Y_{\text {best }}$ indicates the optimal solution seen hitherto regarding the quality of the fitness. At last, the novel position $\mathrm{Y}_{\mathrm{i}, \mathrm{j}+\mathrm{l}}$ is stated in eq. (27). Fig 3 exhibits the flowchart of the proposed model.

$$
\mathrm{Y}_{\mathrm{i}, \mathrm{j}+1}=\mathrm{Y}_{\mathrm{i}, \mathrm{j}}+\mathrm{LF}
$$




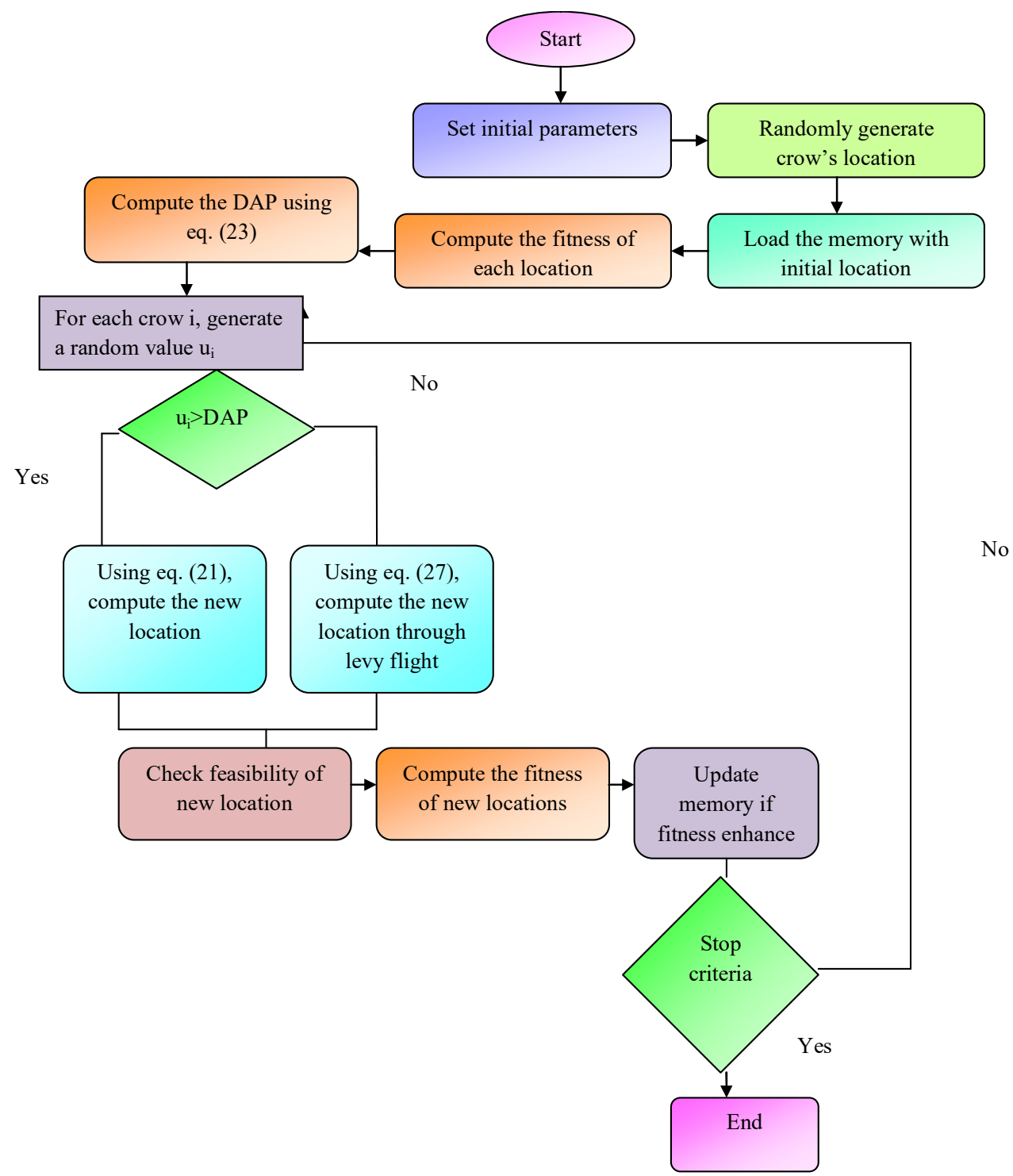

Fig. 3. Flowchart of the proposed model

\section{Result Analysis}

\subsection{Experimental Procedure}

The developed DR detection model has experimented on MATLAB 2014 a. On the HRF dataset, the developed model is analyzed and tested. Here, the High-resolution fundus image is exploited that comprises a total of 30 images, in that 15 images are normal and abnormal, which is downloaded from https://www5.cs.fau. de/research/data/fundus images/. The performance measures like accuracy, specificity, and precision, NPV, sensitivity, F1-score, FDR, MCC, FPR, and FNR were evaluated.

\subsection{Performance Analysis}

Fig. 4 shows the overall classification analysis of the proposed model is shown. Here, the performance of the proposed algorithm is 33\% better than the PSO-NN model, 35\% better than the FF-NN model, and $37 \%$ better than the CS-NN model in terms of the accuracy. Moreover, the proposed method is $12 \%$ better than the PSO-NN model, $16 \%$ better than the FF-NN model, and $18 \%$ better than the CS-NN model in terms of the FNR. In terms of MCC, the proposed method is $12 \%, 13 \%$ and $15 \%$ better than the PSO-NN, FF-NN and CS-NN models. Hence, the improvement in classification has been attained using the proposed model. 


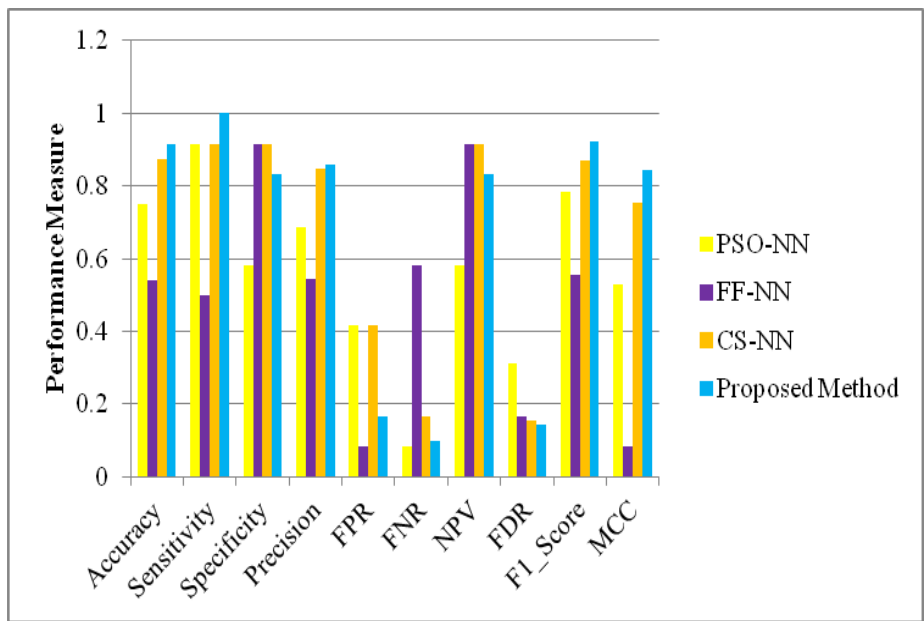

Fig. 4. Performance analysis of the proposed model

\section{Conclusion}

In this paper, ECS-LF algorithm for DR recognition was proposed. Moreover, the proposed model was done in two-phases (i.e.,) DR recognition and blood vessel segmentation. Here, the proposed vessel segmentation algorithm was carried out by three phases. At the first phase, by reconstruction two thresholded binary images were obtained by exploiting tophat and HPF. The areas similar to the two images were obtained as the major vessels, and the remaining pixels in both binary images were integrated to shape a vessel sub-image, which was facilitated to a classification of GMM. As a result, all the pixels in the sub-image that was classified as vessels and amalgamated with the main vessels to obtain the vasculature segmentation. For that reason, the GLRM and GLCM features were extracted from the blood vessels segmentation that was subsequently classified by exploiting NN. Moreover, to widen the accuracy, training was performed by ECS-LF algorithm, with the objective of reducing the error between actual and predicted output.

\section{Compliance with Ethical Standards}

Conflicts of interest: Authors declared that they have no conflict of interest.

Human participants: The conducted research follows the ethical standards and the authors ensured that they have not conducted any studies with human participants or animals.

\section{References}

[1] Asieh Naderi, Reza Zahed, Leila Aghajanpour, Fahimeh Asadi Amoli, Alireza Lashay,"Long term features of diabetic retinopathy in streptozotocin-induced diabetic Wistar rats", Experimental Eye Research, Volume 184, July 2019, Pages 213-220.

[2] Arul J. Duraisamy, Ghulam Mohammad, Renu A. Kowluru,"Mitochondrial fusion and maintenance of mitochondrial homeostasis in diabetic retinopathy", Biochimica et Biophysica Acta (BBA) - Molecular Basis of Disease, Volume 1865, Issue 6, 1 June 2019, Pages 1617-1626.

[3] Ugur Gurlevik, Yasemin Ozdamar Erol, Erdogan Yasar,"Serum and vitreous resistin levels in patients with proliferative diabetic retinopathy Diabetes Research and Clinical Practice, Volume 155, September 2019.

[4] T. Shanthi, R. S. Sabeenian,"Modified Alexnet architecture for classification of diabetic retinopathy images", Computers \& Electrical Engineering, Volume 76, June 2019, Pages 56-64.

[5] X. Zeng, H. Chen, Y. Luo and W. Ye, "Automated Diabetic Retinopathy Detection Based on Binocular SiameseLike Convolutional Neural Network," IEEE Access, vol. 7, pp. 30744-30753, 2019.

[6] Y. Sun, "The Neural Network of One-Dimensional Convolution-An Example of the Diagnosis of Diabetic Retinopathy," IEEE Access, vol. 7, pp. 69657-69666, 2019.

[7] P. Soille, Morphological Image Analysis - Principle and Applications, Springer,Germany, 2003.

[8] Sina Khanmohammadi, Chun-An Chou, "A Gaussian Mixture Model Based Discretization Algorithm for Associative ClassiPcation of Medical Data", Department of Systems Science and Industrial Engineering, 26 March 2016.

[9] Dhanashree Gadkari, "Image Quality Analysis using GLCM", 2004 
[10] Manavalan Radhakrishnan and Thangavel Kuttiannan, "Comparative Analysis of Feature Extraction Methods for the Classification of Prostate Cancer from TRUS Medical Images", IJCSI International Journal of Computer Science Issues, Vol. 9, no.1, January 2012.

[11] Yogeswaran Mohan, Sia Seng Chee, Donica Kan Pei Xin and Lee Poh Foong, "Artificial Neural Network for Classification of Depressive and Normal in EEG", 2016 IEEE EMBS Conference on Biomedical Engineering and Sciences (IECBES), 2016.

[12] Askarzadeh, A. A novel metaheuristic method for solving constrained engineering optimization problems: Crow search algorithm. Comput. Struct. 2016, 169, 1-12.

[13] Emery, N.J.; Clayton, N.S. The Mentality of Crows: Convergent Evolution of Intelligence in Corvids and Apes. Science 2004, 306, 1903-1907.

[14] Holzhaider, J.C.; Hunt, G.R.; Gray, R.D. Social learning in New Caledonian crows. Learn. Behav. 2010, 38, 206219.

[15] N. Chakrabarty, "A Deep Learning Method for the detection of Diabetic Retinopathy," 2018 5th IEEE Uttar Pradesh Section International Conference on Electrical, Electronics and Computer Engineering (UPCON), Gorakhpur, 2018, pp. 1-5.

[16] A. L. Nanayakkara, N. D. Kodikara, A. S. Karunananda and M. M. Dissanayake, "Classification of stages of diabetic retinopathy in human retina," 2016 Sixteenth International Conference on Advances in ICT for Emerging Regions (ICTer), Negombo, 2016, pp. 322-322.

[17] Remmiya R and Abisha C,"Artifacts Removal in EEG Signal Using a NARX Model Based CS Learning Algorithm",Multimedia Research, Volume 1, Issue 1, October 2018.

[18] Ninu preetha and Praveena S,"Multiple Feature Sets and SVM Classifier for the Detection of Diabetic Retinopathy Using Retinal Images ",Multimedia Research, Volume 1, Issue 1, October 2018.

[19] Baronchelli, A.; Radicchi, F. Lévy flights in human behavior and cognition. Chaos Solitons Fractals 2013, $56,101-105$.

[20] Yang, X.-S.; Ting, T.O.; Karamanoglu, M. Random Walks, Lévy Flights, Markov Chains and Metaheuristic Optimization; Springer: Dordrecht, The Netherlands, 2013; pp. 1055-1064.

[21] Yang, X.-S. Nature-Inspired Metaheuristic Algorithms; Luniver Press: Beckington, UK, 2010.

[22] Karaboga, D.; Basturk, B. A powerful and efficient algorithm for numerical function optimization: Artificial bee colony (ABC) algorithm. J. Glob. Optim. 2007, 39, 459-471.

[23] Abràmoff, M., Garvin, M. and Sonka, M., 2010. Retinal imaging and image analysis. Biomedical Engineering, IEEE Reviews in, 3, pp.169--208. 